\title{
Integración de los mensajes comerciales y el contenido editorial en la prensa digital: publicidad nativa
}

\author{
Lidia Maestro Espínola ${ }^{1}$; David Cordón Benito²; Natalia Abuín Vences ${ }^{3}$
}

Recibido: 19 de septiembre de 2018/ Aceptado: 01 de noviembre de 2018

Resumen. Ante un consumidor hiperestimulado por los impactos de las marcas, la publicidad nativa surge como formato integrador de mensajes comerciales y contenido editorial. A través de una revisión documental de las principales investigaciones académicas e informes profesionales, este artículo analiza el concepto de publicidad nativa, ahonda en sus ventajas y muestra las sinergias surgidas entre cabeceras de prensa digitales y anunciantes para beneficiarse de esta nueva herramienta. Las principales conclusiones señalan una mayor eficacia de este tipo de publicidad además de un incremento del engagement con el consumidor y su satisfacción con contenidos de base comercial pero no intrusivos. Palabras clave: publicidad nativa; generación de marca; soporte publicitario; engagement; prensa digital.

\section{[en] Integration of commercial messages and editorial content: native advertising.}

\begin{abstract}
In the face of a hyperstimulated consumer due to the impacts of brands, native advertising emerges as an integrating format for commercial messages and editorial content. Through a documentary review of the main academic research and professional reports, this article analyzes the concept of native advertising, delves into its advantages and shows the synergies that emerged between digital press headers and advertisers to benefit from this new tool. The main conclusions point to a greater effectiveness of this type of advertising as well as an increase in engagement with the consumer and satisfaction with commercially-based but non-intrusive content.
\end{abstract}

Keywords: native advertising; branding; advertising support; engagement; digital press.

Sumario. 1. Introducción. 2. Metodología. 3. El uso de publicidad nativa en empresas editoriales. Casos de éxito. 4. Adaptación de los medios a la publicidad nativa. 5. Eficacia de la publicidad nativa. 6. Publicidad nativa en redes sociales. 7. Conclusiones. 8. Referencia

Cómo citar: Maestro Espínola, L.; Cordón Benito, D.; Abuín Vences, N. (2019). Integración de los mensajes comerciales y el contenido editorial en la prensa digital: publicidad nativa. Revista Pensar en la Publicidad, 13, 209-226.

1 Universidad International de La Rioja. Profesor Contratado Doctor de la Universidad International de La Rioja. E-mail: lidia.maestro@unir.net

2 Universidad Internacional de La Rioja. Profesor Ayudante Doctor de la Universidad International de La Rioja. E-mail: david.cordon@unir.net

3 Universidad Complutense de Madrid. Profesor Contratado Doctor de la Universidad Complutense de Madrid. E-mail: nabuinve@ucm.es 


\section{Introducción}

En los últimos años hemos asistido a una nueva manera de hacer publicidad. Con base en los cambios tecnológicos, variaciones en el consumo de medios, limitaciones económicas de los anunciantes, posibilidades de innovación y, en definitiva, necesidades de incrementar la creatividad y eficacia de los mensajes, se ha impuesto con fuerza el concepto de integración. La publicidad actualmente busca atraer la atención de nuevos consumidores (Health, 2012 ${ }^{4}$ ). Paralelamente a la transformación del sector publicitario, los soportes tienen que adaptarse para dar respuesta a las necesidades de integración que reclaman las estrategias globales de marketing y comunicación.

El desarrollo de estas estrategias tiene como telón de fondo el hecho de que la atención de los lectores hacia los mensajes publicitarios disminuye de manera paulatina. La principal causa apunta hacia un entorno de hiperestimulación cognitiva favorecido por las nuevas tecnologías (Klingberg, 20095). El usuario se ha convertido en un experto en el uso de nuevas técnicas que le permiten filtrar los mensajes publicitarios a través de bloqueadores de publicidad digital. La otra cara de la moneda muestra el interés de los anunciantes por impactar en sus audiencias. Ante esta situación se hace necesario el cambio de herramientas de promoción online que suponen una parte integral de la comunicación. Las agencias de publicidad, en función de los objetivos de los anunciantes, ponen en marcha diferentes estrategias según la eficacia que requieren en cada momento. Los usuarios de las plataformas digitales se han convertido en palancas de cambio en el mercado publicitario y, por ello, las marcas necesitan generar experiencias construidas sobre sus propios valores identitarios.

Durante estas transformaciones el papel de los soportes es fundamental, puesto que las decisiones sobre la oferta de espacios publicitarios influyen en la efectividad de los mensajes. Actualmente, cuando se habla de medios de comunicación se puede hacer referencia a los diferentes canales de distribución de contenidos que no son independientes, sino que están relacionados de manera constante (Añaños y Valli, $2012^{6}$ ). Dentro de ellos se han incorporado los formatos que responden a las demandas que requiere el consumo de medios actual. Esto pasa con los contenidos editoriales y comerciales. Las nuevas audiencias utilizan los medios para informarse, pero, sobre todo, para entretenerse, con una actitud de confianza en unos contenidos que están al servicio del disfrute y no de la persuasión (Shrum, 20047; Nabi y Beth, 2009 ${ }^{8}$; Sayre y King, $2010^{9}$ ). Las estrategias y los ejemplos de campañas integradas contemplan estas necesidades dentro de su planificación, por lo que tienden hacia el uso de diferentes medios, convencionales y no convencionales, con el fin de desarrollar una nueva manera de comunicar mensajes publicitarios y conseguir esa integración de estrategias.

4 Heath, R. (2012): Seducing the Subconscious. The Psychology of Emotional Influence in Advertising, Oxford, Inglaterra, John Wiley \& Sons.

5 Klingberg, T. (2009): The Overflowing Brain: Information Overload and the Limits of Working Memory, Nueva York, Estados Unidos, Oxford University Press.

6 Añaños, E., y Valli, A. (2012): «La publicidad integrada en el contenido TV: Atención visual y reconocimiento cognitivo en los jóvenes y en los adultos mayores [Integrated Advertising in TV content: visual attention and cognitive recognition in young and in older adults]», Pensar la Publicidad, 6(1), 139-162.

7 Shrum, L.J. (Ed.). (2004). The Psychology of Entertainment Media: Blurring the Lines between Entertainment and Persuasion, Mahwah NJ, Taylor \& Francis

8 Nabi, R. y Beth, M. (2009): The SAGE of Media Processes and Effects, Thousand Oaks, Sage.

9 Sayre, S. y King, C. (2010): Entertainment and Society: Influences, Impacts, and Innovations, New York, Routledge. 
Siguiendo esta filosofía de integración de la publicidad y los contenidos, el formato que mejor se adapta a esas nuevas necesidades es el conocido como publicidad nativa. Para acercarse al objeto de estudio esta investigación se centra, por un lado, en el estudio de la eficacia de la publicidad nativa y, por otro, en analizar si las estrategias que la prensa está incorporando se alinean con dichos indicadores de integración.

\section{Metodología}

Este trabajo pone su foco de atención sobre los formatos de publicidad integrada que incluye actualmente la prensa. Por su particular transformación digital y por la necesidad de optimizar las cabeceras digitales, la prensa representa un objeto de estudio para la observación. El objetivo principal de este trabajo es analizar el papel de la prensa digital como soporte publicitario, conocer cómo incorpora estas nuevas estrategias para conseguir una serie de propósitos: en primer lugar, aumentar la eficacia de sus campañas; en segundo lugar, proveer a sus lectores de la información que demandan y, por último, conseguir revalorizar sus espacios.

La metodología que se utiliza para esta investigación tiene un carácter cualitativo, que parte de una revisión bibliográfica del concepto de publicidad nativa y su incorporación como formato publicitario a la prensa digital. Posteriormente, se analizan diferentes casos de éxitos y se aportan los datos del sector sobre la integración de este formato y su eficacia dentro de la prensa digital.

\section{Una aproximación teórica al concepto de publicidad nativa}

El sector de la publicidad y los medios de comunicación se han vinculado para poder transportar los mensajes publicitarios y conseguir la financiación de la actividad periodística. Ante los cambios estructurales en la publicidad y la continua reducción de los ingresos publicitarios es probable que esta vía de financiación no sea suficiente para que las salas de redacción realicen contribuciones importantes a las sociedades democráticas (Siegert, $2013^{10}$ ). Ante la incertidumbre económica, los medios anhelan nuevas entradas de recursos que ayuden a paliar las pérdidas (Maestro et al., $2016^{11}$ ). Medios como Narratively han apostado por eliminar publicidad gráfica tradicional y abrazar el patrocinio de marcas realizando eventos y publicidad nativa (Dowling, 2016 ${ }^{12}$ ). El debate y la preocupación sobre la sostenibilidad económica refleja los enigmas éticos de la incorporación de dichos eventos patrocinados y de la publicidad nativa (Pérez, 2016 ${ }^{13}$ ).

10 Siegert, G. (2013): «From "the end of advertising as we know it" to "beyond content"?». Journalism and media convergence, $5,29$.

11 Maestro Espínola, L. García Santamaría, J.V. y Pérez Serrano, M.J. (2016): «La 'prensa’ digital generalista, como soporte publicitario y los cambios en su modelo de negocio», Revista Latina de Comunicación Social, 71, 1.048 a 1.068 .

12 Dowling, D. (2016): «The business of slow journalism: Deep storytelling's alternative economies», Digital Journalism, 4(4), 530-546.

13 Pérez, F. S. (2016): «Prospective journalism redux: The new life of political magazines in the digital age». International Journal of Media \& Cultural Politics, 12(3), 357-375. 
Los problemas éticos que aporta la inserción de estos formatos publicitarios es un constante objeto de estudio para otros autores que coinciden en los peligros de difuminar la diferencia entre el contenido y la publicidad en la prensa (Macnamara, $\left.2016^{14}\right)$. En concreto, se señala que los anunciantes actúan como intrusos que debilitan la viabilidad de construir una comunidad dentro de una cabecera (Zarzosa y Fischbach, 2017 15 ).

Para abordar el problema ético, es adecuado partir del propio concepto de publicidad nativa. Las marcas han tenido tradicionalmente una obsesión por formar parte de los contenidos editoriales de los medios, y esta idea se ha materializado en el product placement, patrocinio, advertorial o publicidad nativa. Desde una visión académica, se entiende por publicidad nativa cualquier «publicidad pagada que adopta la forma específica y la apariencia del contenido editorial del propio editor» (Wojdynski y Evans, 2016, p.157 ${ }^{16}$ ). La publicidad nativa también se define como una forma de medios pagados donde el contenido comercial se integra dentro del diseño y la forma de contenido editorial, como un intento de recrear la experiencia del usuario al leer noticias en lugar de contenido publicitario (Ferrer Conill, $2016^{17}$ ). Wang y Li ${ }^{18}$ inciden en que se encuentra en la intersección de la publicidad estratégica y la información periodística como un tipo de «contenido híbrido comercial» (2016, p.913). Parece claro que la publicidad nativa es una forma sutil de publicidad digital que se integra estrechamente con su contexto (Harms et al, 2017 ${ }^{19}$ ).

Sharetrough $\left(2015^{20}\right)$ concibe la publicidad nativa como un formato publicitario dentro de una estrategia publicitaria que busca transmitir al usuario un mensaje publicitario que no lo parece y se integra en la estética siguiendo la forma y experiencia del sitio donde está ubicado.

Desde el punto de vista de la industria, el Interactive Advertising Boureau $\left(2017^{21}\right)$ define la publicidad nativa como aquella que se integra en el contenido editorial natural de la página o en la funcionalidad del medio en el que se publica, permitiendo a la marca estar presente en la publicación (sea una web de noticias, un producto audiovisual, un post en un blog especializado...) de una manera más armonizada con el resto de contenido que otros sistemas publicitarios (pop ups, banners y anuncios en general) que puedan aparecer en la web en cuestión. No obstante, el concepto de publicidad nativa no está armonizado a nivel internacional y se puede encontrar que,

14 Macnamara, J. (2016): «The continuing convergence of journalism and PR: New insights for ethical practice from a three-country study of senior practitioners». Journalism \& Mass Communication Quarterly, 93(1), 118141.

15 Zarzosa, J., y Fischbach, S. (2017): «Native Advertising: Trickery or Technique? An Ethics Project and Debate». Marketing Education Review, 27(2), 104-108.

16 Wojdynski, B. W., y Evans, N. J. (2016): «Going native: Effects of disclosure position and language on the recognition and evaluation of online native advertising». Journal of Advertising, 45(2), 157-168.

17 Ferrer Conill, R. (2016): «Camouflaging Church as State: An exploratory study of journalism's native advertising», Journalism Studies, 17(7), 904-914.

18 Wang, Y. y Li, Y. (2017): «Understanding «Native Advertising» from the Perspective of Communication Strategies». Journal of Promotion Management, 23(6), 913-929.

19 Harms, B., Bijmolt, T. H. y Hoekstra, J. C. (2017): «Digital native advertising: practitioner perspectives and a research agenda», Journal of Interactive Advertising, 1-12.

20 Sharethrouh (2015): Native Adertising insgh. http://www.sharethrough.com/nativeadvertising/. Web visitada el $05 / 11 / 2017$.

21 Interactive Advertising Boureau (2017): I Estudio Native and Content Advertising. Recurso electrónico. Recuperado en: http://iabspain.es/wp-content/uploads/estudio-content-native-advertising-2017-vcorta.pdf. Web visitada el 10/02/2018. 
en ocasiones, se entiende como publicidad nativa la publicidad pagada donde el anuncio coincide con la forma, la sensación y la función del contenido de los medios en los que aparece (Native Advertising Institute, 201522).

Actualmente, en línea con esta misma idea, existen numerosos ejemplos que se visualizan diariamente, pero, una vez analizado el concepto, conviene conocer los diferentes tipos de publicidad nativa. Sin embargo, antes de proceder a definir formatos concretos, se cree necesario clasificarlos en dos grades tipologías:

- Anuncios nativos cerrados: aquellos diseñados para promover y anunciar contenidos dentro de una plataforma cerrada, de manera que el usuario no salga de ese entorno si decide visitar el anuncio. Un ejemplo claro de esta tipología serían los tweets promocionados.

- Anuncios nativos abiertos: este tipo de anuncios permiten a las marcas lanzar su campaña de publicidad nativa en múltiples medios al mismo tiempo, de manera que la publicidad se despliega en numerosos espacios.

Descendiendo al detalle, se pueden encontrar los siguientes formatos de publicidad nativa:

- Anuncios In-feed: son los formatos publicitarios que se integran en el timeline de las diferentes redes sociales, mezclándose con el resto de publicaciones promovidas por la red de contactos del usuario que recibe el impacto publicitario.

- Anuncios search de pago. Los pioneros de la publicidad nativa fueron los buscadores, incorporando las búsquedas patrocinadas. Los enlaces patrocinados en los buscadores son un claro ejemplo de publicidad nativa, pues se integran con los resultados de búsqueda y solo se diferencian por su ubicación (antes de los resultados naturales) y su color. Es necesario resaltar que algunos autores y organizaciones descartan que este tipo de anuncios sean considerados publicidad nativa, al no entender los buscadores como un medio propiamente dicho.

- Widgets de recomendación: se trata de pequeñas aplicaciones del anunciante, ubicadas en la homepage del medio o en cualquiera de los espacios que componen el site y que redirigen al usuario a una landing page creada por el anunciante.

- Listados promocionados: este tipo de publicidad nativa no suele aparecer en páginas de contenido editorial, sino que es más común en buscadores, aplicaciones o portales de comercio electrónico. En este caso los productos, bajo el epígrafe de contenido promocionado, se muestran de la misma forma que el resto de productos que se venden en el espacio dentro del que se anuncian.

- Anuncios integrados en el contenido: se trata de formatos que se integran a la perfección en la línea editorial de los medios en los que se publican, adoptando la misma forma que el resto de contenidos que se ofrecen. Se trata de formatos similares a los publirreportajes de la prensa tradicional y deben atraer el interés y la atención de los usuarios del mismo modo que lo hacen los contenidos editoriales.

22 Interactive Advertising Boureau (2015): Guía Legal Sobre Publicidad Nativa. Recurso electrónico. Recuperado en: http://www.iabspain.net/wp-content/uploads/downloads/2015/05/Guia_Legal_IAB_Publicidad_Nativa. pdf. Web visitada el 01/11/2017. 
Según algunas investigaciones, el problema de la publicidad nativa surge cuando existe ausencia de una revelación clara que indique que el mensaje es un anuncio, ya que los medios de comunicación y el anunciante bloquean el conocimiento de persuasión del potencial consumidor. La propia motivación de los formatos nativos consiste en disminuir el conocimiento de defensa y persuasión del espectador (Taylor, $2017^{23}$ ), pero esto puede derivar en problemas éticos.

Parece fundamental la regulación teniendo en cuenta los peligros de una normativa restrictiva, como aborda Hardy $\left(2017^{24}\right)$ desde la perspectiva de la economía política de los medios y la separación de funciones editoriales de la publicidad. Sin embargo, la propia esencia del formato digital complica esta división (Carlson, 2015 ${ }^{25}$ ).

El análisis de este tipo de publicidad capta, cada día, más atención desde el entorno académico. Investigaciones sobre la capacidad de los espectadores para identificar los mensajes de publicidad nativa y los mensajes y el análisis del contexto en el que se insertan son cada vez más comunes. Las investigaciones realizadas apuntan a que la identificación de formatos nativos usando la etiqueta de publicidad o patrocinado aumentan el reconocimiento pero generan evaluaciones negativas (Wojdynski \& Evans, $2016^{26}$ ) que disminuyen las opiniones positivas sobre el editor y la institución publicitada (Amazeen y Wojdynski, 201827; Amazeen y Muddiman, 201728). Por el contrario, la investigación realizada por Jiang et al. $\left(2017^{29}\right)$ aporta resultados que indican que el reconocimiento de publicidad nativa no siempre genera percepciones negativas. Otro experimento de laboratorio que realiza un seguimiento ocular muestra que se produce un mayor reconocimiento publicitario cuando los mensajes están integrados en el texto de la historia que cuando están en la parte superior de la página (Wodjdynski et al, 201730). A su vez, se han realizado investigaciones que profundizan en el reconocimiento de la divulgación y la presencia de la marca en la persuasión de los lectores (Krouwer y Paulussen, 2017'11).

Ante el cambio de paradigma la publicidad y los medios se adaptan y mediante la puesta en marcha de estrategias de comunicación integrada funcionan y logran superar los objetivos (Carretero Pérez y Rangel Velasco, 2017²). Las publicacio-

23 Taylor, C. R. (2017): «Native Advertising: The Black Sheep of the Marketing Family», International Journal of Advertising, 36:2, 207-209.

24 Hardy, J. (2017): «Money,(Co) Production and Power: The contribution of critical political economy to digital journalism studies», Digital Journalism, 5(1), 1-25.

25 Carlson, M. (2015): «When news sites go native: Redefining the advertising-editorial divide in response to native advertising», Journalism, 16(7), 849-865.

26 Wojdynski, B. W., y Evans, N. J. (2016), op.cit., 4.

27 Amazeen, M. A. Y Wojdynski, B. W. (2018): «The effects of disclosure format on native advertising recognicions and audience perceptions of legacy and online news publishers», Journalism, 1464884918754829.

28 Amazeen, M. A. Y Muddiman, A. R. (2017): «Saving Media or Trading on Trust? The effects of native advertising on audience perceptions of legacy and online news publishers», Digital Journalism, 1-20.

29 Jiang, M., Mckay, B. A., Richards, J. I., Y Snyder, W. (2017): «Now You See Me, But You Don’t Know: Consumer Processing of Native Advertisements in Online News Sites», Journal of Interactive Advertising, 17:2, pages $92-108$.

30 Wojdynski, B. W., Bang, H., Keib, K., Jefferson, B. N., Choi, D., Y Malson, J. L. (2017): «Building a Better Native Advertising Disclosure». Journal of Interactive Advertising, 1-12.

31 Krouwer, S., Poels, K. Y Paulussen, S. (2017): «To disguise or to disclose? The influence of disclosure recognition and brand presence on readers' responses towards native advertisements in online news media», Journal of Interactive Advertising, (just-accepted), 00-00.

32 Carretero Velasco, M. J. Y Rangel Pérez, C. (2017): «Comunicación estratégica integral frente a publicidad tradicional». aDResearch ESIC: International Journal of Communication Research/Revista Internacional de Investigación en Comunicación, 15(15). 
nes digitales se pueden convertir en punto de encuentro de lectores que formen una comunidad mientras que la intromisión publicitaria en ellas disminuye el valor informativo para los seguidores (Camarero, Rodríguez Pinto y San José Cabezudo, 201833).

En los últimos años la industria de los medios se ha enfrentado a cambios en la producción, el consumo y la distribución mientras se continúa buscando un modelo de negocio viable. Se ponen en marcha nuevas estrategias que buscan atraer al público. En este sentido las redes sociales obligan a mostrarse de forma diferente ante los lectores, desarrollando informaciones diferentes para consumos diversos con un acceso abierto. Tampoco debe olvidarse que las marcas también se comunican directa e inmediatamente con sus consumidores (Martín-Guart y Fernández-Cavia, 2014 ${ }^{34}$ ). En este entorno la publicidad también se reinventa y varios ejemplos relevantes de ello son los proyectos de publicidad nativa que encontramos en The New York Times, The Washington Post o The Wall Street Journal.

\section{El uso de publicidad nativa en empresas editoriales. Casos de éxito}

Debido a la presión económica, la publicidad nativa se ha convertido en un nuevo modelo de ingresos para los medios que crece hasta convertirse en una «gran tendencia» (Wu et al, 2016, p.1492 ${ }^{35}$ ). Dentro del sector editorial se puede indicar el comienzo de un fenómeno específico, desde que en 2009 Forbes comprase una startup que posteriormente integró dentro de sus procesos editoriales, lo que le llevó a ocupar un lugar reservado hasta entonces a los anunciantes. Uno de los primeros formatos que utilizó consistió en una serie de artículos sobre los inconvenientes del teletrabajo, que firmaba la empresa de bases de datos que acababan de comprar. Durante ese mismo año, Forbes Media anunció la oferta Special Forbes que permitía a los anunciantes tener acceso a una multiplataforma desde la que se les ofrecía la posibilidad de incluir vídeos, infografías, etc. Cabe destacar que la compañía Dell fue la primera en utilizar este servicio.

The Huffington Post fue el siguiente medio norteamericano en poner en marcha esta técnica a través de un acuerdo con Neftlix. Con la publicación de una lista de lugares donde no querrías estar el Día de Acción de Gracias se incluían enlaces que dirigían al usuario a la página de registro de los Servicios de Neftlix. Esta última compañía se ha convertido en una de las empresas que desarrolla estrategias de publicidad integrada con el uso de estos formatos nativos. Otro ejemplo destacable es el éxito obtenido por la campaña diseñada para la serie Orange is the new Black que apareció publicada en el diario americano The New York Times. En agosto de 2014 se publicó un artículo de periodismo de investigación que versaba acerca de los problemas a los que se enfrentan las mujeres en prisión y en el que, además, se

33 Camarero, C., Rodríguez-Pinto, J. Y San José-Cabezudo, R. (2018): «Latent communities of digital publications: The role of editors, followers, and advertisers», Telematics and Informatics

34 Martín-Guart, R. F. Y Fernández Cavia, J. (2014): «La publicidad y la agencia de medios frente al cambio en el ecosistema mediático. [Advertising and media agency against the change in the media ecosystem]». Cuadernos. info, (34), 13-25.

35 Wu, M., Huang, Y., Li, R., Bortree, D. S., Yang, F., Xiao, A., Y Wang, R. (2016): «A tale of two sources in native advertising: Examining the effects of source credibility and priming on content, organizations, and media evaluations». American Behavioral Scientist, 60(12), 1492-1509. 
hacía mención a una serie de reformas necesarias en el código penitenciario estadounidense. Dicho artículo resultó ser un anuncio financiado por los productores de la serie. Cada día son más los medios de comunicación que incorporan estrategias integradas a través del uso de publicidad nativa para rentabilizar sus espacios publicitarios.

Otro de los éxitos de The New York Times ha sido la campaña desarrollada por la compañía Dell en la que se hablaba sobre tecnología. Dentro de la estructura de The New York Times existe un equipo de trabajadores del medio denominados Times Content Studio que se dedican a proponer y buscar anunciantes de valor que ofrezcan una relación bidireccional enriquecedora. Dentro de los medios nativos digitales destaca Buzzfeed por su capacidad para borrar la frontera entre publicidad e información. Entre las diversas estrategias integradas y del uso de publicidad nativa destaca el formato denominado listicle. Las listas son un éxito seguro que proporciona un nivel de confort, seguridad, entretenimiento, interés y constancia al lector. Buzzfeed es un editor social que publica contenido patrocinado sin relación alguna con el producto e introduce publicidad en piezas pequeñas.

Además de publicidad nativa, los medios de comunicación utilizan herramientas 2.0 que les permiten adaptarse a las necesidades del mercado. The Washington Post comenzó a utilizar Clavis para recomendar a sus lectores los artículos que les pueden interesar en base a palabras clave y a los historiales de lectura. Esta iniciativa tiene por nombre The Post Recommends y consiste en un sistema de recomendaciones similar al que utiliza Amazon (cuyo propietario, Jezz Bezos, también compró el Post en 2013).

\section{Adaptación de los medios a la publicidad nativa}

Uno de los objetivos principales de la publicidad nativa es integrar los mensajes publicitarios dentro de la experiencia que el usuario percibe del medio. Se trata de que la función comercial se realice a través de un contenido informativo que le permita interactuar y mejorar el engagement para optimizar la percepción de la marca y fidelizar al público. El término engagement se ha estudiado en diferentes disciplinas como señalan los estudios de Rodgers y Thorson $\left(2018^{36}\right)$ o Aguilera, Baños y Ramirez $\left(2016^{37}\right)$. Estos últimos autores sintetizan dicho término en la «conexión que los clientes, los prospectos y los potenciales forman con determinadas organizaciones a partir de las experiencias que han tenido con las ofertas y actividades de dichas organizaciones».

El consumidor está en primer plano, como ya afirmaba Hopkins $\left(1927,1923^{38}\right)$ o, de manera mucho más reciente, Bassat $\left(1993^{39}\right)$ al inicio de la era digital. Ahora, además, el consumidor tiene a su alcance diferentes técnicas de selección que le permiten bloquear y filtrar impactos. La publicidad nativa, por tanto, viene motivada

36 Rodgers, S. Y Thorson, E. (2018): Special Issue Introduction: Digital Engagement with Advertising. Journal of Advertising, $47: 1,1-3$.

37 De Aguilera, J., Baños, M. Y Perdiguero, J. R. (2016): Consumer engagement. TELOS-Fundación Teléfonica (107).

38 Hopkins, C. C., Lobo, Á. G. Y Bravo, J. (1980): Publicidad cientifica, España, Eresma.

39 Bassat L. (1993): El libro rojo de la Publicidad. Madrid, Debolsillo. 
por este cambio de plano en la importancia del consumidor y por la necesidad de ofrecerle lo que demanda sin interferir, sin que se perciba como una molestia.

Son varias las ventajas que presenta la publicidad nativa: puede llevarse a cabo en cualquier plataforma, permite evitar la saturación que supone un riesgo añadido en el mundo publicitario y, por último, posibilita la segmentación del público. La Guía Legal sobre publicidad Nativa de IAB Spain reclama la importancia que tiene esta segmentación a la hora de causar confusión en grupos de población inexpertos o en acciones dirigidas a menores que no puedan diferenciar que un contenido es publicidad nativa. La normativa legal es muy restrictiva en este aspecto. Según $L a$ Guía Legal sobre Publicidad Nativa (IAB, 201740) la principal obligación de este tipo de mensajes comerciales es la transparencia: deben indicar que se trata de publicidad de una marca determinada. En caso de no cumplir con esta obligación se estaría realizando publicidad encubierta (como tipo específico de publicidad engañosa), por lo que resulta esencial que se identifique claramente que el contenido es publicitario.

Teniendo en cuenta la normativa legal sobre el etiquetado de este tipo de contenidos, según el I Estudio de Efectividad de publicidad nativa en España $\left(2014^{41}\right)$ los medios generalistas son los que más utilizan estas herramientas. Les siguen, en menor proporción, los sites de nichos verticales con presencia social y por último, los portales tradicionales.

Gráfico 1. Ranking de soportes por uso de Publicidad Nativa

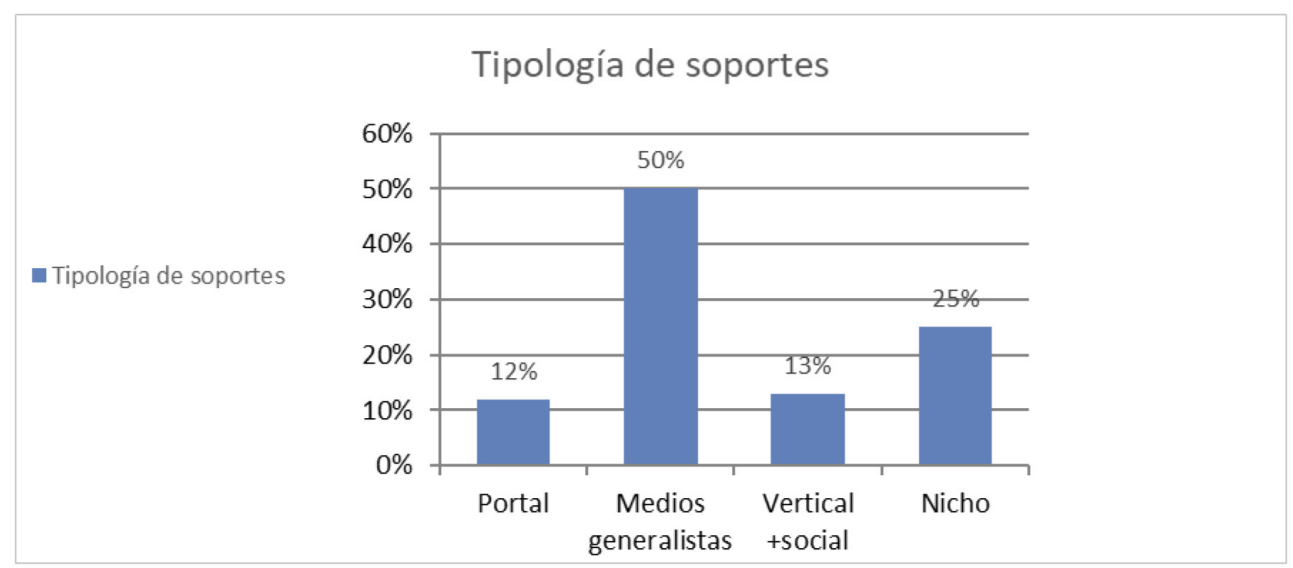

Fuente: Elaboración propia con datos de NativeAD, 2014.

Otra de las conclusiones interesantes del estudio de NativeAD es que los usuarios se implican más en este tipo de contenidos en medios nichos que, a su vez, proporcionan mayor engagement que el que se puede alcanzar a través de los medios que tradicionalmente tienen más lectores. 


\section{Eficacia de la publicidad nativa}

Los objetivos principales de los anunciantes que invierten en publicidad nativa son: aumentar las ventas, reducir costes dentro de la estrategia de marketing o crear una imagen de marca. Es muy habitual que este tipo de iniciativas estén ligadas a la renovación de la imagen de marca y a la asociación con la innovación. Las campañas analizadas para este trabajo representan perfectamente estas teorías.

IPG Media Lab ha realizado un estudio para la revista Forbes que confirma que la imagen que los usuarios tienen de una marca mejora considerablemente cuando sufren el impacto de una campaña de publicidad nativa que cuando lo sufren a través de un banner tradicional. Afirman que quienes ven un anuncio de publicidad nativa tienen un $41 \%$ más de probabilidades de adquirir un producto o servicio de la empresa anunciante. Si se focaliza la atención sobre el engagement que se genera, esta mejora dentro de las redes sociales. El estudio identifica que el usuario comparte con más facilidad un artículo perteneciente a un medio de prestigio que el de una web de empresa.

Otra de las claves del éxito de la publicidad nativa radica en el contenido. El hecho de aparecer junto a noticias reales y hacer hincapié sobre el valor editorial sin utilizar palabras promocionales pone en valor estos espacios. Los lectores de diarios digitales buscan información y entretenimiento Las campañas con más éxito son las que hacen referencia a este tipo de contenidos.

Para el correcto diseño de la publicidad nativa hay que perseguir los siguientes objetivos esenciales dentro de nuestra campaña:

1. Adaptación: de los contenidos de la marca a la experiencia que identifica al medio editorial. El contenido debe personalizarse, estar adaptado a todos los dispositivos e integrado en el contexto del soporte.

2. Plataforma: la elección del soporte donde se va a desarrollar tiene que seleccionarse en función de los objetivos y el público final, pero cuidar de manera especial su alineación con los contenidos. Estos deben adaptarse en función de la plataforma en la que se publiquen.

3. Certificación de contenido: todo anuncio nativo tiene que etiquetarse de manera que el usuario siempre sepa que lo que está viendo es publicidad. El art. 26 de la Ley de Competencia Desleal prohíbe que se pueda «incluir como información en los medios de comunicación, comunicaciones para promocionar un bien o servicio, pagando el empresario o profesional por dicha promoción, sin que quede claramente especificado en el contenido o mediante imágenes y sonidos claramente identificables para el consumidor o usuario que se trata de un contenido publicitario».

4. Engagement: nos permite conocer el interés y la relación que los consumidores tienen con una marca. Mide el éxito de una compañía por sus estrategias y ayuda a calcular el retorno de inversión, el coste de conseguir un consumidor.

El contenido es la pieza fundamental para el éxito de la publicidad nativa. Permite dar confianza al consumidor, pero tiene que ser relevante para el lector y ofrecer diferenciación y presencia para el soporte en el que se aloja. Esta técnica sigue el principio ético de señalar su vinculación comercial para no engañar al consumidor, 
pero este hecho no desmotiva al lector. Sharethrough $\left(2015^{42}\right)$ confirma que los usuarios miran un $2 \%$ más el contenido nativo que el editorial. Esto se traduce en una ventaja exitosa que aumenta el recuerdo positivo y la participación activa del consumidor.

Por otro lado, la IAB $\left(2017^{43}\right)$ ha hecho recientemente un experimento en el que se exponía a una muestra de usuarios a una serie de creatividades digitales. Algunas de ellas respondían a los patrones de la publicidad digital tradicional: banners, skin... y otros correspondían a la modalidad de publicidad nativa. Posteriormente se les realizaron una serie de cuestiones dirigidas a analizar la percepción sobre ambos tipos de publicidad, obteniendo los siguientes resultados:

Tabla 1. Percepción de los diferentes tipos de publicidad

\begin{tabular}{|l|l|l|}
\hline Publicidad Tradicional & & Publicidad Nativa \\
\hline $\mathbf{2 9} \%$ & Contenido relacionado con la marca/producto & $\mathbf{5 1 \%}(+22 \%)$ \\
\hline $\mathbf{4 1 \%}$ & Orientada al consumidor/personalizada & $\mathbf{5 2 \%}(+11 \%)$ \\
\hline $\mathbf{3 5 \%}$ & Transmite los valores de la marca/producto & $\mathbf{4 1 \%}(+6 \%)$ \\
\hline $\mathbf{2 2} \%$ & Hace que me sienta identificado con el contenido & $\mathbf{2 5 \%}(+3 \%)$ \\
\hline
\end{tabular}

Fuente: I Estudio Native and Content Advertising, IAB (2017)

Tal y como se puede observar, la investigación revela que más de la mitad de los sujetos objeto de estudio perciben los formatos nativos como contenido relacionado con la marca/ producto y consideran que están orientados al consumidor. También opinan que transmite en mayor medida los valores de la marca/producto y obtiene mejores resultados que la publicidad tradicional en conseguir la identificación del usuario con el contenido. Este mismo estudio ha revelado que el $94 \%$ de los usuarios que consume alguno de los formatos de la publicidad nativa ve cubiertas sus expectativas.

Así mismo, se testó la opinión y percepción de los anunciantes respecto este tipo de formatos, revelando los siguientes datos:

- 6 de cada 10 anunciantes declaran que, en combinación con la publicidad tradicional, mejora los resultados y es una alternativa interesante a la saturación que sufre el mercado.

- Un tercio de los profesionales coinciden en que permite acceder a targets nicho, que impacta de forma más efectiva a los jóvenes y que es un formato adecuado para transmitir los valores de una marca.

- De forma minoritaria, indican que este tipo de formatos aseguran una mayor notoriedad de marca y que incrementan la cobertura de campaña.

\footnotetext{
42 Sharethrouh (2015): Native Adertising insgh. http://www.sharethrough.com/nativeadvertising/. Web visitada el $05 / 11 / 2017$.

43 Interactive Advertising Boureau (2015): op. cit., 4
} 
- Así mismo, un 66\% de los anunciantes encuestados asegura que incrementarán la partida presupuestaria en estos formatos para el año 2018.

También hay que señalar como Gerard Baker, editor jefe de The Wall Street Journal explica en una entrevista recogida por campaignasia.com, que la publicidad nativa puede afectar a la confianza del consumidor negativamente ya que «la gente confía en nosotros y tenemos que tener cuidado. Hay que ser capaces de que el lector no encuentre problema para diferenciar los artículos y editoriales de la publicidad nativa».

El formato ayuda a la identificación de este tipo de publicidad, aunque, como marca la normativa, este tipo de contenido siempre tiene que aparecer etiquetado ${ }^{44}$. Será importante conocer los formatos más utilizados para analizar las estrategias de publicidad nativa.

Gráfico 2. Formatos de publicidad nativa más usados

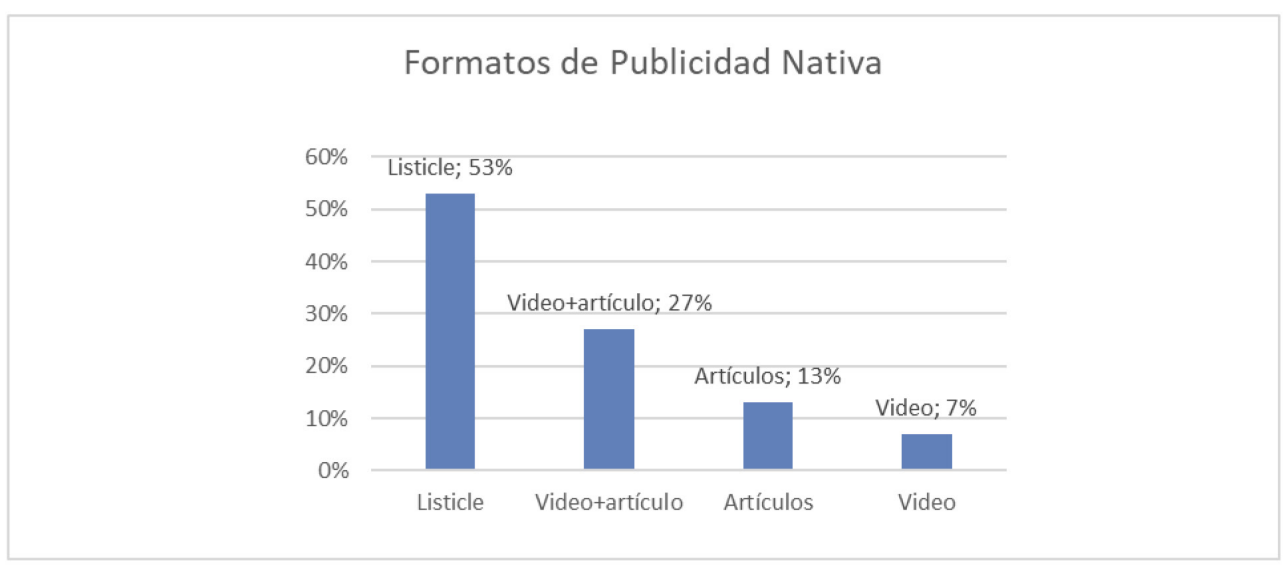

Fuente: Elaboración propia con datos de NativeAD ${ }^{45}, 2014$

El engagement que se consigue guarda relación con los diversos formatos que se utilizan. Los objetivos de cada campaña determinan la selección de formatos que es conveniente utilizar.

Como se puede observar, las lecturas o artículos son el formato que más engagement generan, y eso se debe a que son el que mejor se integra con el contenido. Estos datos permiten afirmar que, a mayor integración, más eficaz es el formato nativo que utilicemos.

44 Hay tres requisitos para el etiquetado de Publicidad nativa: correctamente etiquetado con un tamaño suficiente para que el usuario lo advierta y agrupado al contenido de referencia, texto que describa la condición de contenido pagado y la relación con el usuario en cuanto a la privacidad y protección de datos, se ofrece un canal de comunicación al usuario.

45 Native Ad (2015): op. cit., 8. 
Gráfico 3. Engagement por formato de Publicidad Nativa

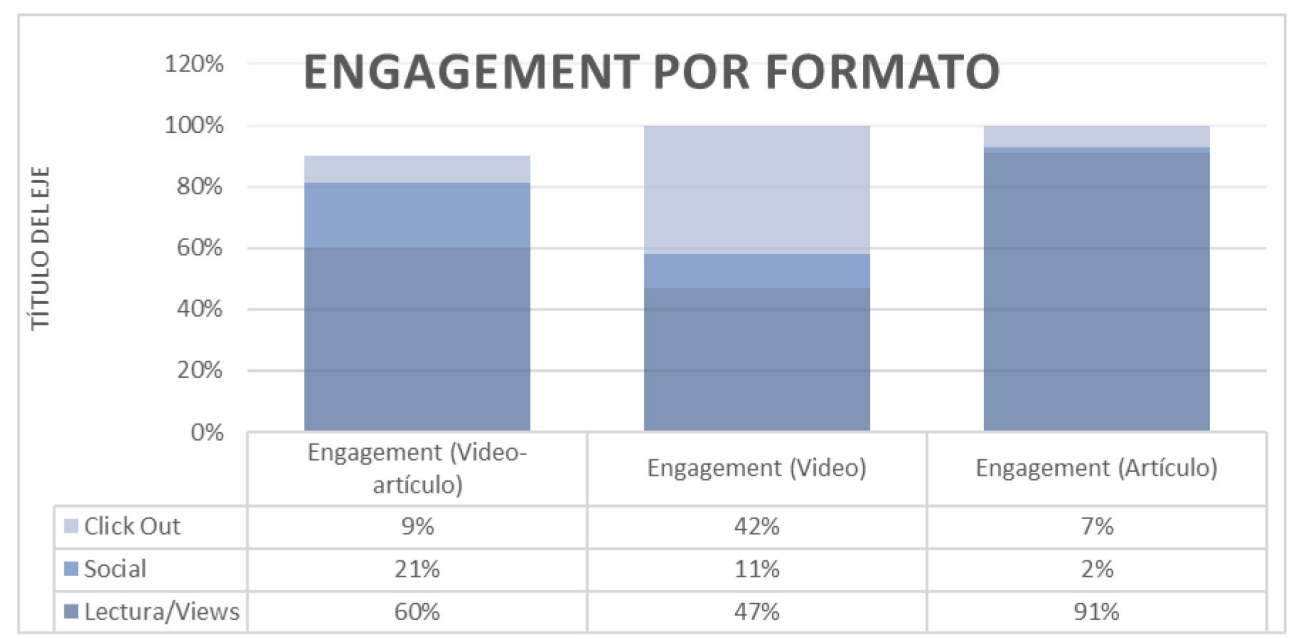

Fuente: Elaboración propia con datos de NativeAd, 2014.

\section{Publicidad nativa en redes sociales}

Las redes sociales experimentan el mayor crecimiento en cuanto a inversión publicitaria presentándose como servicios consolidados que han propiciado la publicidad 2.0 (Beelen, 2006 ${ }^{46}$ ). Twitter recibe más de 320 millones de usuarios al mes y Facebook más de 500 millones de visitas mensuales. Las campañas alojadas permiten la interacción y comunicación directa con los consumidores posibilitando una mayor fidelización. Los formatos de publicidad tradicional no funcionan bien en este entorno, por lo que los anunciantes invierten cada vez más en publicidad nativa. Podemos confirmar que la publicidad integrada «discurre por la intersección entre la potente expansión de las nuevas redes digitales y la progresiva fragmentación de los medios tradicionales, que todavía ostentan la hegemonía» (Corredor, 2010 $0^{47}$ ).

Según BI Intelligence $e^{48}$ se estima que entre los años 2013 y 2018 los anuncios de publicidad nativa que se incluyan en redes sociales obtendrán la mayor cuota de mercado alcanzando los 12 millones de dólares. La inversión en publicidad nativa engloba formatos display, contenido patrocinado y redes sociales.

Según las previsiones que se realizan para este campo es necesario analizar el papel de los medios en las redes sociales que va a permitir realizar conclusiones sobre las estrategias que se están llevando a cabo.

46 Beelen, P. (2006): «Publicidad 2.0». http://www.paulbeelen.com. http://www.paulbeelen.com/whitepaper/Publicidad20.pdf. Web visitada el 03/04/2018.

47 Corredor, P. (2010): «Bajo el signo de la integración: creatividad, innovación y tecnología al servicio de la publicidad en la era digital,. Telos: Cuadernos de comunicación e innovación, (82), 65-77.

48 Bi Intelligence (2015): «Spending on native advertising is soaring as marketers and digital media publishers realize the benefits» http://www.businessinsider.com/spending-on-native-ads-will-soar-as-publishers-and-advertisers-take-notice-2014-11. Web visitada el 22/02/2018. 
Gráfico 4. Previsión de la Inversión de Publicidad Nativa 2013-2018. EE.UU.

(en millones de dólares)

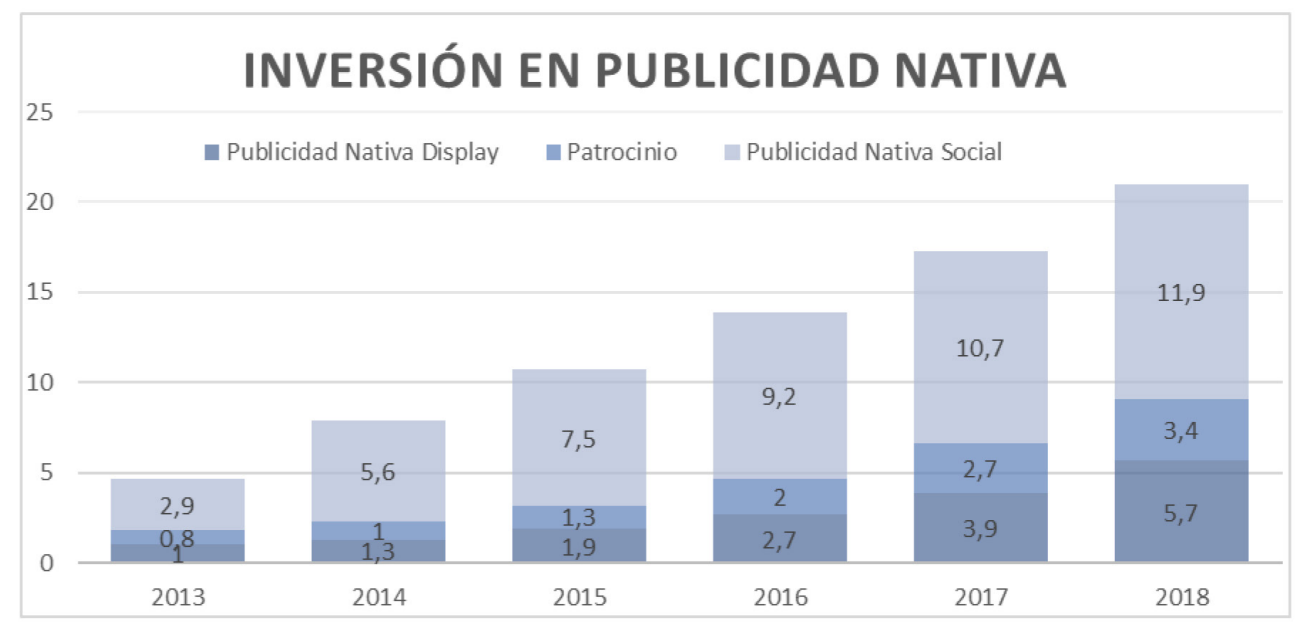

Fuente: Elaboración propia con datos de BI Intelligence

Si la atención se centra en la eficacia de las campañas nativas en redes sociales el I Estudio de Eficacia de Publicidad Nativa de NativeAd ${ }^{49}$ confirma que el canal que consigue más engagement social es Facebook con un $88 \%$ y Twitter con un $12 \%$.

\subsection{Engagement}

Para realizar un correcto análisis de efectividad debe plantearse la ratio de engage$m e n t^{50}$ que posee cada soporte. La investigación realizada por NativeAd indica que los medios generalistas son los que más utilizan este tipo de publicidad. Además, destaca que la ratio de conversión mayor pertenece a los medios que son nicho. La ratio de engagement es ligeramente inferior entre los medios generalistas y los nichos, pero se compensa con el volumen tan elevado de usuarios.

Las campañas tienen que mantener una presencia en todos los canales y las redes sociales son una parte imprescindible para las estrategias integradas.

El I Estudio de Eficacia de Publicidad Nativa de NativeAd extrae importantes conclusiones para analizar las estrategias de Publicidad integrada que se pueden encontrar en los medios. Confirma diferentes ratios de conversión (CTR $)^{51}$ : un $2 \%$ de CTR para la publicidad nativa insertada en un destacado de la sección noticia y un $7 \%$ en los formatos de vídeo que se presenta al total de lectores. Se planifican un $50 \%$ de las campañas dentro de los medios generalistas, seguidos de los medios nicho con un $25 \%$. El nivel de efectividad mayor lo ostentan las campañas que incluyen un mix de soportes en medios y vertical social. Todos los datos y campañas

\footnotetext{
$49 \quad$ Native Ad (2015): op. cit., 11

50 Ratio de engagement: número de visitas dividido entre el total de consumos de contenido

51 Ratio de conversión: número de impresiones/ total visitas a página de contenido.
} 
analizados confirman que los lectores no rechazan la publicidad nativa y que, incluso, mantienen una ratio de consumo similar al del resto de contenidos editoriales.

\section{Conclusiones}

Tras un exhaustivo análisis de las principales investigaciones e informes profesionales publicados hasta la fecha, tanto a nivel nacional como internacional, podemos afirmar que la publicidad nativa supone una segunda oportunidad para la publicidad digital por los siguientes motivos:

- La eficacia de la publicidad display está muy mermada, debido fundamentalmente a la saturación publicitaria que sufre internet, que hiperestimula al usuario. Estos formatos poseen características formales y estructurales muy similares, por lo que los usuarios han aprendido a reconocerlos y los obvian de una manera inconsciente. La eficacia de la publicidad display es muy escasa, y la integración que ofrece la publicidad nativa es la única oportunidad de conseguir resultados satisfactorios, pues los estudios revelan que posee una mayor capacidad para atraer la atención del potencial consumidor.

- Internet y las redes sociales han modificado la forma en que nos relacionamos. Permite una mayor participación y el usuario ya no quiere ser un consumidor pasivo de contenidos si no que quiere participar del proceso comunicativo. Desde el punto de vista publicitario, esto se traduce en la necesidad de ofrecer al potencial consumidor experiencias que vayan más allá del simple mensaje comercial que apela a la emoción.

- Los diferentes estudios llevados a cabo hasta la fecha, revelan que la publicidad nativa genera una mayor implicación del potencial consumidor con los contenidos comerciales. Así mismo, se puede afirmar que cuando se toman decisiones siguiendo una estrategia integrada, como es el caso de los formatos de publicidad nativa, se enriquece la lectura o visualización del anuncio. Son una fuente de ingresos para los soportes que, al mantener una continuidad en la experiencia de usuario, agregan valor a la campaña y al medio. El lector disfruta de contenidos con una base publicitaria sin estar sometido a las continuas interrupciones que percibe como molestia. No solo es una alternativa al display tradicional, sino que, más bien, se transforma en un complemento de valor.

- Una de las causas del bajo rendimiento de la publicidad display es que entorpece la navegación y molesta al usuario, además de desperdiciar muchos impactos. La publicidad nativa respeta al lector durante su navegación y consigue un impacto mayor sobre un público más segmentado. Este hecho, junto a que los contenidos que se presentan apuestan por una calidad editorial que les aporta valor, provocan que los lectores mejoren su atención en un $2 \%$ más sobre el contenido nativo que sobre el editorial. La continuidad y la presencia en otros canales como YouTube o redes sociales como Facebook y Twitter aumentan la fidelidad, el recuerdo positivo de la marca y la participación activa del lector.

- El contenido es una pieza clave que fideliza al lector. Sin embargo, esta calidad del contenido debe enriquecerse e integrar todos los canales a disposición de las cabeceras. Para conseguir esto, las estrategias empleadas deben 
diseñarse para unificarse y reforzarse a través de la distribución por medio de otros canales. De manera tradicional la publicidad compite con el contenido informativo del que se hacen eco los medios de comunicación y en los formatos integrados esta constante se mantiene, por lo que el contenido se convierte en la clave del éxito.

No obstante, además de ventajas la publicidad nativa también posee inconvenientes y entraña algunos peligros que hay que tener en cuenta:

- Uno de los problemas reseñados en las principales investigaciones está relacionado con la ética. Al tratarse de publicidad integrada y que adopta un formato similar al contenido editorial, se corre el riesgo de que el usuario la tome por información. Desde el punto de vista legal debe aparecer claramente identificada, para evitar que sea catalogada como publicidad encubierta. Así mismo, el hecho de hacerla pasar por contenido editorial, puede generar percepciones negativas en el receptor al pensar que se trata de un engaño del anunciante.

- Otro de los problemas con los que se encuentra la publicidad nativa es el temor de los medios de asociar sus contenidos editoriales a este tipo de anuncios. Sin embargo, los análisis del sector revelan que este tipo de formatos generan mejores percepciones entre los potenciales consumidores que los anuncios digitales convencionales.

Las empresas de prensa generalista mantienen su fortaleza debido, entre otros motivos, a los contenidos que publican, por lo que las estrategias publicitarias de este tipo pueden encontrar una relación fructífera alineada en su misma referencia de valor.

\section{Referencias}

Amazeen, M. A. Y Muddiman, A. R. (2017): «Saving Media or Trading on Trust? The effects of native advertising on audience perceptions of legacy and online news publishers», Digital Journalism, 1-20.

Amazeen, M. A. Y Wojdynski, B. W. (2018): «The effects of disclosure format on native advertising recognicions and audience perceptions of legacy and online news publishers», Journalism, 1464884918754829.

Añaños, E., \& Valli, A. (2012): «La publicidad integrada en el contenido TV: Atención visual y reconocimiento cognitivo en los jóvenes y en los adultos mayores [Integrated Advertising in TV content: visual attention and cognitive recognition in young and in older adults]», Pensar la Publicidad, 6(1), 139-162.

Bassat L. (1993): El libro rojo de la Publicidad. Madrid, Debolsillo.

Beelen, P. (2006): «Publicidad 2.0». http://www.paulbeelen.com. http://www.paulbeelen. com/whitepaper/Publicidad20.pdf. Web visitada el 03/04/2018.

Bi Intelligence (2015): «Spending on native advertising is soaring as marketers and digital media publishers realize the benefits» http://www.businessinsider.com/spending-on-native-ads-will-soar-as-publishers-and-advertisers-take-notice-2014-11. Web visitada el 22/02/2018. 
Camarero, C., Rodríguez-Pinto, J. Y San José-Cabezudo, R. (2018): «Latent communities of digital publications: The role of editors, followers, and advertisers», Telematics and Informatics.

Carlson, M. (2015): «When news sites go native: Redefining the advertising-editorial divide in response to native advertising», Journalism, 16(7), 849-865.

Carretero Velasco, M. J. Y Rangel Pérez, C. (2017): «Comunicación estratégica integral frente a publicidad tradicional». aDResearch ESIC: International Journal of Communication Research/Revista Internacional de Investigación en Comunicación, 15(15).

Corredor, P. (2010): «Bajo el signo de la integración: creatividad, innovación y tecnología al servicio de la publicidad en la era digital, Telos: Cuadernos de comunicación e innovación, (82), 65-77.

De Aguilera, J., Baños, M. Y Perdiguero, J. R. (2016): Consumer engagement. TELOS-Fundación Teléfonica (107).

Dowling, D. (2016): «The business of slow journalism: Deep storytelling's alternative economies», Digital Journalism, 4(4), 530-546.

Hardy, J. (2017): «Money,(Co) Production and Power: The contribution of critical political economy to digital journalism studies», Digital Journalism, 5(1), 1-25.

Harms, B., Bijmolt, T. H. Y Hoekstra, J. C. (2017): «Digital native advertising: practitioner perspectives and a research agenda», Journal of Interactive Advertising, 1-12.

Heath, R. (2012): Seducing the Subconscious. The Psychology of Emotional Influence in Advertising, Oxford, Inglaterra, John Wiley \& Sons.

Hopkins, C. C., Lobo, Á. G. Y Bravo, J. (1980): Publicidad cientifica, España, Eresma.

Interactive Advertising Boureau (2017): I Estudio Native and Content Advertising. Recurso electrónico. Recuperado en: http://iabspain.es/wp-content/uploads/estudio-content-native-advertising-2017-vcorta.pdf. Web visitada el 10/02/2018.

Interactive Advertising Boureau (2015): Guía Legal Sobre Publicidad Nativa. Recurso electrónico. Recuperado en: http:/www.iabspain.net/wp-content/uploads/downloads/2015/05/Guia_Legal_IAB_Publicidad_Nativa.pdf. Web visitada el 01/11/2017.

Jiang, M., Mckay, B. A., Richards, J. I., Y Snyder, W. (2017): «Now You See Me, But You Don't Know: Consumer Processing of Native Advertisements in Online News Sites», Journal of Interactive Advertising, 17:2, pages 92-108.

Klingberg, T. (2009): The Overflowing Brain: Information Overload and the Limits of Working Memory, Nueva York, Estados Unidos, Oxford University Press.

Krouwer, S., Poels, K. Y Paulussen, S. (2017): «To disguise or to disclose? The influence of disclosure recognition and brand presence on readers' responses towards native advertisements in online news media», Journal of Interactive Advertising, (just-accepted), 0000 .

Maestro Espínola, L. García Santamaría, J.v. Y Pérez Serrano, M.J. (2016): «La 'prensa' digital generalista, como soporte publicitario y los cambios en su modelo de negocio», Revista Latina de Comunicación Social, 71, 1.048 a 1.068.

Macnamara, J. (2016): «The continuing convergence of journalism and PR: New insights for ethical practice from a three-country study of senior practitioners». Journalism \& Mass Communication Quarterly, 93(1), 118-141.

Martín-Guart, R. F. Y Fernández Cavia, J. (2014): «La publicidad y la agencia de medios frente al cambio en el ecosistema mediático. [Advertising and media agency against the change in the media ecosystem]». Cuadernos.info, (34), 13-25.

Nabi, R. Y Beth, M. (2009): The SAGE of Media Processes and Effects, Thousand Oaks, Sage. 
Native Ad (2015): I Estudio de Eficacia de Publicidad Nativa. http://www.elpublicista.es/ adjuntos/fichero_10140_20150109.pdf. Web visitada el 07/05/2017.

Pérez, F. S. (2016): «Prospective journalism redux: The new life of political magazines in the digital age». International Journal of Media \& Cultural Politics, 12(3), 357-375.

Rodgers, S. Y Thorson, E. (2018): Special Issue Introduction: Digital Engagement with Advertising. Journal of Advertising, $47: 1,1-3$.

Sayre, S. Y King, C. (2010): Entertainment and Society: Influences, Impacts, and Innovations, New York, Routledge.

Sharethrouh (2015): Native Adertising insgh. http://www.sharethrough.com/nativeadvertising/. Web visitada el 05/11/2017.

Shrum, L.J. (Ed.). (2004). The Psychology of Entertainment Media: Blurring the Lines between Entertainment and Persuasion, Mahwah NJ, Taylor \& Francis.

Siegert, G. (2013): «From «the end of advertising as we know it» to «beyond content?». Journalism and media convergence, 5, 29.

Taylor, C. R. (2017): «Native Advertising: The Black Sheep of the Marketing Family», International Journal of Advertising, 36:2, 207-209.

Wang, Y. Y Li, Y. (2017): «Understanding «Native Advertising» from the Perspective of Communication Strategies». Journal of Promotion Management, 23(6), 913-929.

Wojdynski, B. W., Bang, H., Keib, K., Jefferson, B. N., Choi, D., Y Malson, J. L. (2017): «Building a Better Native Advertising Disclosure». Journal of Interactive Advertising, $1-12$.

Wojdynski, B. W., Y Evans, N. J. (2016): «Going native: Effects of disclosure position and language on the recognition and evaluation of online native advertising». Journal of Advertising, 45(2), 157-168.

Wu, M., Huang, Y., Li, R., Bortree, D. S., Yang, F., Xiao, A., Y Wang, R. (2016): «A tale of two sources in native advertising: Examining the effects of source credibility and priming on content, organizations, and media evaluations». American Behavioral Scientist, 60(12), 1492-1509.

Zarzosa, J., Y Fischbach, S. (2017): «Native Advertising: Trickery or Technique? An Ethics Project and Debate». Marketing Education Review, 27(2), 104-108. 